\title{
A specific arousal effect from barpressing on nonspecific arousal in rats
}

\author{
JOHN H. GILL and HAROLD C. NIELSON \\ University of Utah, Salt Lake City, Utah 84112
}

\begin{abstract}
Running-wheel activity and barpressing on either an FR 10 or FI 30-sec schedule of sugarwater reward were recorded, respectively, as measures of nonspecific and specific arousal of rats. The running-wheel activity of all rats was consistent across days and across within-day segments. Also, higher rates of running-wheel activity were associated with higher rates of FR performance but not FI performance. These results showed that nonspecific arousal had a positive relationship with both nonincentive- and incentive-motivated behaviors, although the relationship with the incentive task appeared to depend on whether efficient task performance could be facilitated or not by the higher levels of arousal. A separate effect of specific arousal also was in evidence since higher rates of activity on the running-wheel measure, relative to the previous night, were observed to follow higher rates of FR performance. The findings were interpreted as evidence for separate systems of arousal.
\end{abstract}

Investigators who have attempted to identify relationships between the general activity of animals and their rates of activity under specific incentive motivation generally have referred to "arousal" as a way of describing "how much of" these behaviors occurs. One of the first investigators to be concerned with arousal was Cannon (1929). He believed that arousal was induced when any physiological system either exceeded or was decreased below a homeostatic balance point. The arousal that resulted from this homeostatic imbalance controlled both the particular response and its rate of occurrence. More recently, investigators have tried to specify how a single arousal system might lead to different behaviors. For example, Jacobs and Farel (1971) argued that several motivated behaviors might share a common substrate of arousal while an animal is guided toward specific behavioral sequences by external cues. Bindra $(1969,1974)$ shared a similar viewpoint and postulated a central motive state (arousal) that was generated by both an organismic state (physiological condition) and external stimulation which came from goal objects (incentive stimulation). Other arousal theorists have postulated dualarousal systems. Routtenberg (1968), for example, suggested a system where one source of arousal is concerned primarily with the general activation of responses and another is more specific to the incentive or reward that accompanies behavior. Similarly, Whalen (1966) differentiated "arousability," or the propensity for arousal, from "arousal" that is the current level of excitation modulated by the presence or absence of relevant stimuli.

The purpose of the present experiment was to try to differentiate two individual sources of arousal from the behavior of rats. One source of arousal was viewed as nonspecific and was regarded to have its source in any of a number of tissue needs, hormonal states, or circadian biorhythms. Nonspecific arousal was operationalized as daily running-wheel activity when no specific incentive objects were associated with running. Systematic changes in this activity over the 24-h day have been attributed to circadian periodicities (Richter, 1967). Specific arousal was thought to be derived from incentive-motivated task performance, although this arousal also might have a momentary feedback effect on nonincentive motivated activity. Barpressing on one of two schedules of reinforcement (FR or FI) was the incentive task.

\section{METHOD}

\section{Subjects and Apparatus}

The subjects were 32 adult, male Wistar rats that weighed between 352 and $440 \mathrm{~g}$ at the start of experimental procedures. The rats were housed individually in Wahmann activity wheels in rooms where temperatures were thermostatically maintained at $24^{\circ} \pm 11^{\circ} \mathrm{C}$, with relative humidity of $58 \%-64 \%$, and were maintained on a 12:12-h, light-dark cycle.

For barpressing, the rats were taken to a separate room where they were placed into one of two identical sound-attenuated experimental conditioning chambers. In the center of one wall of each chamber and $5.5 \mathrm{~cm}$ above the floor was a recessed food well into which a dipper full of sugar water (30\% sucrose) was positioned for $4 \mathrm{sec}$. A retractable lever was placed to the left of this food well at a height of $6.5 \mathrm{~cm}$ above the floor and a distance of $9 \mathrm{~cm}$ from the center of the food well. The chambers were programmed to deliver rewards on an FR or FI schedule to either chamber through electromechanical relay circuitry.

\section{Procedure}

During weight stabilization and a period of adjustment to the running wheels, the rats were given ad-lib access to water, but their food was regulated so their body weights gradually were reduced to and maintained at $\mathbf{8 0 \%}$ of their free-feeding weights. 
The rats then were shaped to barpress for sugar water rewards on a CRF schedule. When barpress rates were stable, the rats were divided into two reinforcement schedule groups based on their CRF barpressing rates, with an equal number of fast and slow responders in each group. An attempt also was made to equate the running-wheel activity levels for the two groups. One group of rats then was trained to press the bar on an FR 10 schedule of reinforcement, and the second group pressed the lever on an FI 30-sec schedule. Training continued across 11 weeks, with three daily sessions (training days) occurring each week, with 1 or 2 no-training days separating each training day. The sessions were programmed to last for $5 \mathrm{~min}$ for each of the animals of both reward-schedule groups and were run in the morning hours between 0730 and $0945 \mathrm{~h}$. Groups were counterbalanced between experimental chambers, and the order of running the groups was alternated eack. training day. Daily maintenance procedures that included weighing and feeding the rats occurred during the training periods or at the same time on no-training days. Although the time to complete these procedures was shorter on no-training days, deliberate delays were introduced on no-training days to minimize this difference. The rats were fed at the conclusion of each maintenance or training period.

Daily records were kept of running-wheel activity differentiating three separate periods: Dark-period activity included running-wheel rates from $1800 \mathrm{~h}$ until approximately $0730 \mathrm{~h}$. Maintenance activity included the time from the start of maintenance and leverpressing procedures $(0730 \mathrm{~h})$ until their completion $(0945 \mathrm{~h})$, and lightperiod activity included the time from the end of maintenance procedures to the start of the dark-period activity. For statistical purposes, the total running-wheel activity (wheel revolutions) of each segment was divided by the time duration of that segment so that the average amount of activity per unit of time could be compared across activity segments. In addition, records of total barpresses and reinforcements were kept for each rat. For purposes of this report, only the data from the final 6-day period were used; this included 3 training days separated by 3 notraining days. All comparisons used two-tailed tests.

\section{RESULTS}

Table 1 presents the mean-hourly activity scores of rats on both the FR 10 and the FI 30-sec reward schedules across the final 3 training and final 3 notraining days. Also presented in this table are the Pearson product-moment coefficients that compared these scores.

Table 1

Mean Hourly Absolute Activity Scores and Correlation Coefficients of FR and FI Groups Across the Final 3 Training (T) and Final 3 No-Training (NT) Days

\begin{tabular}{|c|c|c|c|c|}
\hline Measure & Mean & Measure & Mean & $\mathrm{r}$ \\
\hline \multicolumn{5}{|c|}{ FR Group $(n=16)$} \\
\hline Dark, $\mathrm{T}$ & 52.2 & Dark, NT & 83.1 & .90 \\
\hline Maintenance, $\mathrm{T}$ & 521.6 & Maintenance, NT & 536.7 & .95 \\
\hline Light, T & 58.5 & Light, NT & 145.3 & .75 \\
\hline Dark, T & & Light, T & & .70 \\
\hline Dark, NT & & Light, NT & & .86 \\
\hline \multicolumn{5}{|c|}{ FI Group $(n=16)$} \\
\hline Dark, T & 51.3 & Dark, NT & 76.2 & .91 \\
\hline Maintenance, $\mathrm{T}$ & 568.3 & Main tenance, NT & 475.6 & .98 \\
\hline Light, T & 68.2 & Light, NT & 134.1 & .82 \\
\hline Dark, $T$ & & Light, $\mathrm{T}$ & & .74 \\
\hline Dark, NT & & Light, NT & & .73 \\
\hline
\end{tabular}

Note-All Pearson coefficients yielded rs at $p<.01$.
The correlation coefficients showed that the relative activity rates of both reward-schedule groups were stable across training and no-training days and also across within-day segments. Hence, in each instance, rats that ran the most at one time of day consistently ran more at other times during the 24-h period and across the 6 days, irrespective of barpress training.

To evaluate the barpressing data, barpresses were correlated with running-wheel activity. The correlations were statistically significant only for the FR group on light-period measures of both training [ $\mathrm{r}=$ $.55, \mathrm{t}(14)=2.47, \mathrm{p}<.05]$ and no-training days $[r=.52, t(14)=2.45, p<.05]$. These coefficients showed that rats with higher FR barpress rates also had greater daytime running activity scores. Activity measures for the maintenance segments were just short of accepted levels of reliability (ps $<.10$ ), possibly because the rats' activity was disrupted by the barpress and maintenance procedures.

To assess further the relationship between barpress rates and subsequent running-wheel activity, a second measure of running-wheel activity was derived which permitted a comparison of a rat's light-period running-wheel behavior with its own running-wheel behavior of the previous night. Mean-hourly lightperiod measures $(\mathrm{A})$ and the mean-hourly dark-period measures (B) were set in the formula $A /(A+B)$. By this formula, scores above .50 indicated relatively greater hourly activity during the light period and scores below .50 reflected relatively greater hourly activity during the previous dark period. Since the above measures had shown high consistency between light and dark activity, a relatively greater amount of activity following barpressing for rats with higher rates of barpressing should reflect the influence of barpressing, or specific arousal, on that day's runningwheel behavior. No such effect of specific arousal should be observed on the derived running-wheel measures on no-training days. In each of the FR and FI groups, rats were divided into two subgroups based on their median-total barpress scores and their derived activity measures were compared on both training and no-training days. On training days, the eight FR-trained rats that had the highest total barpress rates $(\bar{X}=335.5)$ had higher derived activity scores $(\overline{\mathrm{X}}=.55 \pm .17)$, reflecting greater light-relativeto-dark-period activity, than did the eight rats whose barpress scores were below the median, showing less light-relative-to-dark-period activity $[\overline{\mathrm{X}}$ total barpresses $=139.4, \bar{X}$ derived activity $=.35 \pm .17$; $\mathrm{t}(14)=2.37, \mathrm{p}<.05]$. This difference occurred despite the fact that average hourly activity rates were less following the maintenance periods of training days than of no-training days $[\mathrm{t}(14)=3.65$, $\mathrm{p}<.01$ ]. When derived activity measures were compared for these same rats on no-training days, there was no difference observed, nor were there differences 
on either training or no-training days for rats in the FI group. The overall mean FI barpress rate was 85.3.

\section{DISCUSSION}

Two individual sources of arousal, nonspecific and specific, can be inferred from the present results. First, the measure of nonspecific arousal was runningwheel activity which was observed to be stable both within and across daily segments and also across days when operant training did or did not occur. In addition, higher rates of running-wheel behavior during the light periods of both training and no-training days was associated with higher rates of performance on the FR schedule. These results showed that runningwheel activity, as the measure of nonspecific arousal, was positively related to both nonincentive- and incentive-motivated behaviors. The fact that a relationship with barpressing was not observed for rats in the FI group requires further comment. FR and FI schedules differ in that high rates of activity facilitate FR performances but are counterproductive to efficient FI performances. Hence, it would seem reasonable that nonspecific arousal would have a positive influence on an incentive-motivated task that was facilitated by such arousal, whereas no relationship would be observed when the additional arousal might interfere with efficient task performance.

Specific arousal can be inferred from the feedback effect of FR performances on the derived measure of running-wheel activity. This measure showed that the rats with the higher FR performance rates ran more in the wheels that day than they had the previous night, whereas rats with lower FR performance rates ran less on this measure. This effect did not extend to days when no operant training was given and was not observed on either days for rats in the FI group. Hence, it would appear that specific arousal was generated in the interaction of nonspecific arousal with FR performances and that this specific arousal had a positive effect on the subsequent runningwheel activity of the rats. The fact that no relationship of this type occurred for rats in the FI group again supports the contention that a differential arousal effect had not been generated by an interaction of nonspecific arousal with FI performances.

There are two concerns with the above contention that require further elaboration. One is that different levels of specific arousal in fact may have been produced by nonspecific arousal interacting with FIschedule performance but that the rats learned to suppress this arousal in learning to perform "efficiently." However, given the chance for specific arousal to be "expressed" after barpressing, no enhancement of running-wheel activity was observed. The second concern is that specific arousal may have been generated but was "displaced" into "interim" activities (e.g., grooming) during the interreinforcement intervals. We did not measure interim activities in the present experiment, and so this latter alternative must remain a possibility. However, if differential levels of specific arousal were generated by FI performances, unlike FR performances, they did not extend to the running-wheel activity of that day.

The present findings cannot be interpreted in a single-arousal system where increased amounts of general arousal might be expected from faster barpressing performances. If this had occurred, we would have expected general increases in all activities, since higher nonspecific arousal would have produced higher rates of barpressing that would have fed back onto running-wheel activity in a cumulative fashion. Running-wheel activity actually was less after barpressing than on no-training days. In addition, we also argue against differences in hunger or in incentive delivery as single sources of arousal, since the measures of running-wheel activity that correlated with barpressing occurred after the rats had been fed. However, we do not want to suggest that incentives themselves cannot be a significant source of arousal induction under certain circumstances. Killeen, Hanson, and Osborne (1978), for example, have found that presentation of incentives associated with "prepared" behaviors (Seligman, 1970), or unlearned activity, generates arousal that decays exponentially over time and that this arousal cumulates with shorter reinforcement intervals, thus generating even higher levels of arousal.

The present findings extend the work of Killeen et al. (1978) in that their findings apply specifically to arousal following noncontingent reward delivery, whereas the present results apply to reward delivery that is contingent on a learned or "unprepared" leverpress. Kileen et al. (1978) reported that rats that leverpressed for rewards did not show the arousal effect associated with incentive delivery and suggested that new behaviors or "unprepared" responses may not show susceptibility to induction by heightened arousal levels associated with incentive delivery. On the basis of our findings, we would agree that such arousal induction does not depend on incentive delivery, but does occur dependent on the interaction of nonspecific arousal with performances of certain incentive-motivated tasks. That is, specific arousal induction depends upon whether nonspecific arousal will be associated positively or negatively with efficient performance. This specific arousal then momentarily will feed back onto general activity or onto whatever nonincentive-motivated activity happens to occur.

\section{REFERENCES}

Bindra, D. The interrelated mechanisms of reinforcement and motivation, and the nature of their influence on response. In W. J. Arnold \& D. Levine (Eds.), Nebraska Symposium 
on Motivation (Vol. 17). Lincoln: University of Nebraska Press, 1969.

Bindra, D. A motivational view of learning, performance, anc. behavior modification. Psychological Review, 1974, 81, 199-213.

CAnnon, W. B. Bodily changes in pain, hunger, fear, and rage (2nd ed.). New York: Appleton-Century-Crofts, 1929.

JACOBS, B. L., \& FAREL, P. B. Motivated behaviors produced by increased arousal in the presence of goal objects. Physiology \& Behavior, 1971, 6, 473-476.

Killeen, P. R., Hanson, S. J., \& Osborne, S. R. Arousal: Its genesis and manifestation as response rate. Psychological Review, 1978, 85, 571-581.

Richter, C. P. Psychopathology of periodic behavior in animals and man. In J. Zubin \& H. F. Hunt (Eds.), Comparative psychopathology: Animal and human. New York: Grune and Stratton, 1967.

Routtenberg, A. The two-arousal hypothesis: Reticular formation and limbic system. Psychological Review, 1968, 75, 51-80. .

Seligman, M. E. P. On the generality of the laws of learning. Psychological Review, 1970, 77, 406-418.

Whalen, R. E. Sexual motivation. Psychological Review, 1966, 73, 151-163.

(Received for publication March 1, 1979; revision accepted September 13, 1979.) 